DOI: $10.5433 / 1981-8920.2010 v 15 n 2 p 1$

\title{
COMPETÊNCIA INFORMACIONAL: ANÁLISE EVOLUCIONÁRIA DAS TENDÊNCIAS DA PESQUISA E PRODUTIVIDADE CIENTÍFICA EM ÂMBITO MUNDIAL
}

\section{ALFABETIZACIÓN EN INFORMACIÓN: ANÁLISIS DE LA EVOLUCIÓN DE LAS TENDENCIAS DE LA INVESTIGACIÓN Y LA PRODUCTIVIDAD CIENTÍFICA EN TODO EL MUNDO}

\begin{abstract}
Elisabeth Adriana Dudziak - elisabeth@usp.br Doutora em Engenharia pela Escola Politécnica da Universidade de São Paulo, USP Bibliotecária do Departamento Técnico do Sistema Integrado de Bibliotecas da Universidade de São Paulo
\end{abstract}

\begin{abstract}
Resumo
Objetiva analisar a produtividade científica e identificar tendências de pesquisa em torno do tema da competência informacional (information literacy), tomando por base pesquisa realizada em duas das principais bases de dados internacionais multidisciplinares dedicadas à análise de citações: Web of Science (WoS) e SCOPUS. Inicia abordando o significado da análise bibliométrica e sua importância na determinação de tendências da atividade e produtividade científicas. Apresenta a evolução do conceito de competência informacional, desde sua origem (em 1974) até 2010. Realiza varredura sobre a produção científica registrada na forma de artigos e documentos localizados nas referidas bases utilizando como estratégia de busca a expressão "information literacy". Conclui com análise das tendências mundiais de pesquisa sobre competência em informação.
\end{abstract}

\section{Palavras-chave}

Competência em informação. Produção científica. Análise de citações. Web of Science. SCOPUS. 


\section{INTRODUÇÃO}

Construir um quadro referencial evolutivo das tendências de pesquisa em uma determinada área de conhecimento ou tópico não é tarefa simples. Envolve conhecimento aprofundado da historiografia do assunto que, a rigor, apenas pode dar conta de uma parcela da totalidade do conhecimento produzido na área. A começar pelo necessário recorte e limitação à produção científica veiculada em canais formais de comunicação e informação.

Neste sentido, a cientometria e a bibliometria podem ser muito úteis. Enquanto a cientometria é definida como o estudo da mensuração e quantificação da evolução da ciência, a análise bibliométrica tem sido bastante utilizada como ferramenta de avaliação da produtividade, intensidade e tendências das atividades científicas, bem como na determinação de fluxos de informação e produção de conhecimento a respeito de determinado campo de conhecimento, tópico de pesquisa, grupo de pesquisadores, instituição ou país. Várias métricas têm sido aplicadas no sentido de aumentar a confiabilidade e a efetividade destas análises: índices de citações, ranqueamento de periódicos, fator de impacto, pesquisadores mais proeminentes (fator $\mathrm{H}$ ), número de publicações por país, número de patentes registradas, mapeamento de redes de pesquisa, trajetórias científicas e conceituais.

No campo da Ciência da Informação, o registro da informação e sua influência sobre a comunicação científica em biblioteconomia e ciência da informação (LIS) foi explorada por Silveira e Bazi (2008) e Blessinger e Hrycaj (2010). Nesta perspectiva, destaca-se a importância das análises dos periódicos científicos e seus elementos constitutivos para se "auferir o grau de institucionalização cognitiva e social de uma disciplina científica" (SILVEIRA; BAZI, 2008, p. 11).

Com relação ao tema da competência em informação (information literacy), houve um crescimento exponencial dos estudos e pesquisas publicados em fontes formais de informação, principalmente nos periódicos científicos internacionais, principalmente na última década. Apesar das controvérsias e da relutância por parte de alguns profissionais quanto à adoção do conceito de competência em informação e sua aplicação, observa-se, além do espalhamento geográfico, a diversidade de campos de aplicações e práticas ligadas ao tema, em distintas áreas de conhecimento. Em função desta realidade, e reafirmando sua inequívoca ligação com a ciência da informação, torna-se fundamental analisar tendências e rumos da pesquisa científica sobre competência informacional.

É deste ponto de partida que se estrutura o presente artigo, cujo objetivo é analisar a produtividade científica em torno do tema da competência informacional (information literacy), tomando por referência pesquisa realizada nas bases de dados internacionais e multidisciplinares que contemplam a análise de citações que são a Web of Science e o Scopus. 


\section{REFERENCIAL TEÓRICO}

\subsection{Produtividade Científica}

Observando o processo de validação de novos saberes da ciência, percebe-se que houve uma modificação estrutural no fluxo da informação científica (BARRETO, 1998), uma vez que a comunicação tradicional e impressa deu lugar à informação eletrônica, incorporando novos canais de conhecimento, com destaque para os periódicos.

Neste processo, parâmetros aplicados à ciência e seu universo tornaram-se essenciais na validação e relevância da informação científica divulgada: a avaliação pelos pares, método, credibilidade, verificabilidade, consistência, objetividade, coerência e originalidade.

A publicação dos resultados da pesquisa científica e tecnológica é importante por uma série de fatores: dá visibilidade à pesquisa, possibilita a análise de tendências da ciência e da tecnologia, permite avaliar o desempenho dos pesquisadores, analisar as instituições de ensino e pesquisa por meio de sua produção científica, possibilita avaliar o impacto da pesquisa na comunidade científica, auxilia na definição de normas, políticas e investimentos, torna possível quantificar e qualificar a ciência por meio de métricas e estudos bibliométricos que auxiliam na avaliação da credibilidade de informações, origem dos autores e dos dados, bem como o impacto da pesquisa na comunidade científica a partir do índice de citações.

Segundo Silveira e Bazi (2009, p.1), "O ato de citar representa o movimento que um pesquisador realiza na construção do conhecimento, recorrendo a outros estudos para construir o seu" e, desta forma, também ratifica o valor do conhecimento referenciado a partir da citação das fontes de informação. Neste sentido, ainda de acordo com estes autores, o estudo das citações pode ser feito a partir do uso de métodos quantitativos e qualitativos.

Do ponto de vista quantitativo, as análises se baseiam em parâmetros mensuráveis estruturados sobre valores numéricos que buscam a quantificação da produção científica sobre o tópico e, a partir dela, a avaliação da produção científica de autores e instituições, a partir da mensuração do número de citações de artigos de periódicos e autores, levando em conta recortes geográficos e temporais, entre outros parâmetros. Por outro lado, do ponto de vista qualitativo, as análises recaem sobre os contextos de produção e de divulgação do conhecimento científico, bem como a identificação de determinadas linhas de pensamento, tipologia de estudos, análise de conteúdos, métodos e tendências conceituais gerais subjacentes.

A produtividade dos autores, conhecida também como "lei de Lotka", é considerada uma subárea da bibliometria e também se baseia no índice de citações e redes estabelecidas (ALVARADO, 2009, p.39). Quanto maior a produtividade de um autor, maior será a chance de ser citado. Por outro lado, quanto mais relevante um determinado artigo for para uma área de conhecimento ou tópico específico, tanto maior será o número de citações obtido. 
É dentro deste universo que os estudos bibliométricos surgiram e se fortaleceram. Otlet (1934 apud PINHEIRO, 1983, p. 60) define Bibliometria como a parte da bibliologia que se ocupa da medida ou quantidade aplicada a livros mas, de fato, o termo é atribuído a Pritchard que, em 1969, definiu Bibliometria como modalidade de estudo que aplica métodos matemáticos para quantificar os processos de comunicação escrita, considerando tanto livros como outros meios de comunicação.

A frequência das citações mede a atividade científica em uma determinada área de conhecimento. Além disso, auxilia na determinação de frentes de pesquisa, ajuda na identificação dos autores mais ativos na área, elucida a gênese de determinado conceito, termo ou expressão, bem como o grau de perenidade (meia-vida) dos artigos publicados ao longo dos anos.

A utilização de programas de computador nos estudos bibliométricos tem se popularizado, assim como indicadores de produtividade tornaram-se acessíveis a partir de bases de dados internacionalmente reconhecidas como a Web of Science (WoS). Tais bases agrupam e tornam acessível a "corrente principal" dos periódicos científicos.

Nos periódicos da corrente principal concentra-se a mais alta porcentagem das citações de nível alto e médio. Isto significa que "as chances dos documentos publicados em periódicos da corrente principal serem altamente citados são 18\% mais elevadas do que aqueles publicados em periódicos não pertencentes a essa corrente" (ALVARADO, 2009, p. 52). Outra importante relação se refere ao idioma nos quais os documentos foram publicados e a frequência de citações. Observa-se que o inglês praticamente exerce o monopólio sobre as citações, demonstrando que os artigos publicados neste idioma têm mais chance de serem citados e, de fato, são mais citados, numa relação de 44 para 2, conforme informações de Alvarado (2009, p. 53).

Outro aspecto relevante ligado à Bibliometria diz respeito aos agrupamentos de artigos publicados em periódicos científicos, determinando zonas de produtividade, "onde a primeira zona constitui o núcleo de periódicos mais estreitamente relacionados com a área que está sendo estudada" (PINTO; IGAMI ; BRESSIANE, 2010, p.203). Trata-se da lei de Bradford.

Além destes parâmetros, temos o fator de impacto, que é produto da relação entre os artigos citados e os artigos publicados de um determinado título de periódico em um determinado intervalo temporal. $O$ índice de imediaticidade mede com que rapidez um artigo publicado é imediatamente citado. A meia-vida mede quanto tempo depois de ter sido publicado um artigo ainda é citado. $\mathrm{O}$ índice h mede as citações dos autores em relação às suas publicações. Estes são apenas alguns dos parâmetros atualmente utilizados no ambiente acadêmico cujo objetivo é verificar e direcionar a produtividade científica e acadêmica. 


\subsection{Origem e evolução do conceito de Information Literacy}

Nenhum estudo que pretende revelar tendências atuais das pesquisas sobre determinado tópico pode prescindir de uma análise cuidadosa do núcleo fundador da frente de pesquisa selecionada. Por esta razão, conhecer a origem e a evolução do conceito de information literacy é fundamental na construção do quadro teórico de referência.

A expressão Information Literacy (literalmente traduzida como alfabetização informacional) surgiu pela primeira vez na literatura em novembro de 1974, em um relatório intitulado: The information service environment relationships and priorities ${ }^{1}$, de autoria do bibliotecário americano Paul G. Zurkowski que era, naquele momento, - Presidente da Information Industry Association (IIA), e integrava a equipe da National Commission on Libraries and Information Science. A meta de Zurkowski e sua equipe era estabelecer as diretrizes para um programa nacional de preparação e acesso universal à Information Literacy, que seria concluído até 1984. Este direcionamento teve origem nas constatações feitas por Zurkowski em seu próprio ambiente de trabalho. Seu relatório de 30 páginas, além de sugerir a disseminação de bancos de dados informacionais e a adoção de indicadores, identifica questões políticas relacionadas à informação, a partir das seguintes afirmações:

a) A informação adiciona valor ao país e à população;

b) Provavelmente $100 \%$ da população americana é alfabetizada, mas somente uma pequena porção pode ser considerada alfabetizada em informação;

c) Os recursos informacionais devem ser aplicados às situações de trabalho;

d) Existem inúmeras rotas de acesso e fontes de informação;

e) Estas rotas de acesso e fontes são pouco conhecidas e subutilizadas;

b) Técnicas e habilidades são necessárias no uso das ferramentas de acesso à informação, assim como no uso de fontes primárias;

f) A informação deve ser usada na resolução de problemas;

g) O setor privado necessita de informações para se desenvolver;

h) A relação entre as bibliotecas e as indústrias passa por um momento de transição (ZURKOWSKI, 1974).

Zurkowski (1974) menciona ainda a importância dos indicadores de produtividade científica obtidos no Science Citation Index e no Social Science Citation Index, consideradas importantes fontes de informação desde aquela época. Nos anos que se seguiram, muitos estudiosos passaram a adotar a expressão information literacy, especialmente nos círculos profissionais, ao discutir questões relacionadas ao domínio do universo informacional. Provavelmente, o Relatório de Zurkowski (disponibilizado pelo Education Resources Information Center - ERIC) é o documento mais ci-

1 O relatório de Zurkowski foi recentemente disponibilizado online no seguinte endereço: http://www.eric.ed.gov/PDFS/ED100391.pdf 
tado por toda a comunidade científica dedicada à pesquisa sobre competência informacional ${ }^{2}$.

Em 1976, Burchinal retomaria o assunto através da publicação de seu trabalho intitulado The communications revolution: America's third century challenge. In the future of organizing knowledge, publicado na Assembléia Geral das Bibliotecas Universitárias do Texas, Estados Unidos.

Ainda em 1976, Cees Hamelink, consultor em comunicação de massa nos Estados Unidos, citou o termo referindo-se à necessidade premente do cidadão de libertar-se das imposições e regras criadas pelos sistemas de comunicação e informação e, a partir de uma formação livre de influências ou de informações prédigeridas, adquirir uma perspectiva holística, individual e independente dos acontecimentos (DUDZIAK, 2001). De modo similar, Major R. Owens (1976), bibliotecário norte americano, referiu-se à Information Literacy como elemento essencial à democracia e à constituição da cidadania. Em 1979, Robert S. Taylor, outro bibliotecário norte americano, estabeleceu o vínculo definitivo entre os bibliotecários e a information literacy, em artigo destinado a discutir o futuro da profissão e intitulado Reminiscing about the Future: Professional Education and the Information Environment. Publicado no periódico Library Journal, o artigo apresenta as seis áreas de desenvolvimento profissional e educacional que Taylor considerava como as mais promissoras; destaca, dentre estas áreas, a information literacy.

Em 1981, Paul G. Zurkowski voltaria à cena científica com artigo de sua autoria (publicado no mesmo periódico Library Journal) intitulado The Library Context and the Information Context: Bridging the Theoretical Gap. Neste artigo, faz uma revisão sobre o desenvolvimento histórico das bibliotecas e apresenta a evolução da indústria da informação, ao descrever o impacto da tecnologia de informação sobre as bibliotecas. Até então, nas bibliotecas o que se priorizava era a educação de usuários, primordialmente a partir da orientação de uso das bibliotecas públicas. $\mathrm{Na}$ década de 80 , houve um avanço significativo quando os bibliotecários passaram a adotar a expressão orientação bibliográfica como ação educativa relacionada ao conhecimento das fontes informacionais e ferramentas para localização da informação. Em 1985, o estudo conduzido por Patricia Breivik viria a modificar drasticamente o cenário e o significado da atuação educacional do bibliotecário. A partir do estudo de usuários da Biblioteca da Universidade de Colorado em Denver, E.U.A., a autora constatou que a information literacy seria a principal atividade educacional a ser desenvolvida nas universidades deste momento em diante (DUDZIAK, 2003). As necessidades de aprendizado dos alunos não podiam mais ser satisfeitas com os livros textos e os materiais existentes nas bibliotecas. Era preciso dar a eles condições para que aprendessem mais e melhor, de maneira independente e autônoma.

Corroborando a visão de Breivik (1985), em 1987, a monografia de Carol C. Kuhlthau intitulada Information Skills for an Information Society: a review of research, lançou as bases da Information Literacy Education, ou seja, a Educação voltada para a Alfabetização em Informação, segundo dois eixos fundamentais: sua integração ao

\footnotetext{
${ }^{2}$ O Google Acadêmico registrou em Setembro de 2010 exatas 115 citações.
} 
currículo, a partir da proficiência em investigação, identificada como a meta das bibliotecas do ensino médio; e o amplo acesso aos recursos informacionais, cruciais ao aprendizado estudantil, a partir da apropriação das tecnologias de informação e das ferramentas de busca.

Em 1989, a ALA - American Library Association, publicou o Presidential Committe on Information Literacy: Final Report (Chicago: ALA, 1989), preparado por um grupo de bibliotecários e de educadores, onde foram definidas as bases da information literacy. Largamente reproduzida e disseminada, é hoje um das definições mais citadas na literatura:

Para ser competente em informação, uma pessoa deve ser capaz de reconhecer quando uma informação é necessária e deve ter a habilidade de localizar, avaliar e usar efetivamente a informação. (....) Resumindo, as pessoas competentes em informação são aquelas que aprenderam a aprender. Elas sabem como aprender pois sabem como o conhecimento é organizado, como encontrar a informação e como usá-la de modo que outras pessoas aprendam a partir dela. (ALA, 1989, p.1 $)^{3}$

O relatório da ALA ressaltava a importância da Information Literacy para indivíduos, trabalhadores e cidadãos, reforçando o papel da informação na resolução de problemas e tomada de decisão. As recomendações se concentraram na implantação de um novo modelo de aprendizado, com a diminuição da lacuna existente entre sala de aula e biblioteca. Tal implantação só seria possível a partir de uma reestruturação curricular, onde fosse privilegiado o uso dos recursos informacionais disponíveis, para a aprendizagem e resolução de problemas, de forma contextualizada, a fim de incutir nos aprendizes o hábito de buscar e utilizar a informação (e a biblioteca). A partir desse documento, o conceito se popularizou (DUDZIAK, 2001).

Na segunda metade da década de 80 , os primeiros estudos de caso e surveys relacionados ao tema foram publicados. Na década de 90 surgiram os primeiros livros e as primeiras dissertações e teses sobre o assunto. Somente em 1992, o ERIC incorporou a expressão "information literacy" à sua lista de descritores.

A partir de então, os profissionais da informação, conscientes da necessidade de possibilitar o acesso rápido e fácil ao novo universo informacional, de maneira eficaz e eficiente, voltaram-se para a Information Literacy. Passaram a pensar em tornar os usuários da biblioteca (agora usuários da informação) aprendizes independentes, enfatizando a integração curricular e a cooperação com a comunidade.

3 "To be information literate, a person must be able to recognize when information is needed and have the ability to locate, evaluate, and use effectively the needed information...Ultimately, information literate person are those who have learned how to learn. They know how to learn because they know knowledge is organized, how to find information, and how to use information in such a way that others can learn from them. They are people prepared for lifelong learning, because they can always find the information needed for any task or decision at hand."(ALA, 1989) 
Todo mundo usa informação enquanto cidadão, trabalhador, na resolução de problemas ou para o aprendizado ao longo da vida. Tradicionalmente as escolas promovem o conceito "aprender a aprender". As competências mais elevadas de aprendizado incluem a formulação de questões, a avaliação da informação de acordo com sua pertinência e exatidão, a organização da informação e, finalmente, a aplicação da informação para responder as questões originais - o último e mais valioso passo no processo. Não se trata somente de achar a informação, mas usá-la para motivar 0 aprendiz (DOYLE, 1994, p.1).

A Information Literacy tem suas fundações na Biblioteconomia e Ciência da Informação, mas é fato que a literatura produzida na área nem sempre tem sido unânime no uso e atribuição de significado para a expressão. Algumas vezes, é usada para substituir expressões já conhecidas como a educação de usuários e conceitos subordinados (orientação bibliográfica, treinamento de uso da biblioteca, visita orientada

Enquanto nos Estados Unidos e outros países de língua inglesa o uso da expressão literacy (alfabetização) associada ao termo informação foi largamente aceito, em outros países sua utilização ainda varia. Em Portugal, por exemplo, a expressão foi traduzida como literacia informacional ou literacia da informação. Em países onde se fala o espanhol, adota-se preferencialmente a expressão alfabetización informacional, mas a expressão competência informacional também é utilizada.

No Brasil, a questão da tradução da expressão information literacy ainda suscita discussão e não há consenso. Alguns bibliotecários e pesquisadores da área utilizam a expressão alfabetização informacional, outros adotam letramento informacional, enquanto muitos utilizam competência informacional. Dado que, no país, a alfabetização tem seu significado fortemente associado às fases iniciais da educação, ao passo que a literacia e o letramento ligam-se predominantemente ao universo das palavras, é preciso refletir sobre a terminologia mais adequada e representativa. A adoção da tradução do conceito como competência informacional ou competência em informação parece ser a melhor escolha, por ter significado mais abrangente, além de ser aceita e valorizada tanto na área educacional quanto nos círculos profissionais.

Pessoas competentes em informação estão familiarizadas com as várias mídias de informação, incluindo jornais, revistas, televisão, internet, entre outras. Sabem como o mundo da informação é estruturado, como acessar as redes formais e informais de informação, conhecem as estruturas de comunicação.

A partir do quadro evolutivo das pesquisas sobre information literacy delimitado até aqui, foi possível constatar que a gênese da expressão foi expressa por Zurkowski em 1974. Embora fosse bibliotecário, Zurkowski captou bem o momento de transição no qual as tecnologias de informação criavam uma nova realidade, que desencadearia novas demandas por parte das empresas, do setor industrial e da sociedade em geral. Além de Zurkowski (1974), outros autores 
formam o núcleo fundador das pesquisas: bibliotecários e educadores tais como Burchinal (1976), Hamelink (1976), Taylor (1979), Breivik (1985), Kuhlthau (1987), como também a ALA (1989), profissionais e instituições que ajudaram a pavimentar o caminho inicial das pesquisas sobre o tema.

A seguir, apresenta-se a síntese deste núcleo de autores ligados às pesquisas iniciais sobre o tema, precursores que utilizaram a expressão information literacy (Quadro1).

\begin{tabular}{|c|c|c|}
\hline Autores & Artigos & Datas \\
\hline ZURKOWSKI, P.G. & $\begin{array}{l}\text { Information services environment relationships } \\
\text { and priorities }\end{array}$ & 1974 \\
\hline BURCHINAL, L.G. & $\begin{array}{l}\text { The communications revolution: America's third } \\
\text { century challenge }{ }^{4} \text {. }\end{array}$ & 1976 \\
\hline HAMELINK, C. & An alternative to news & 1976 \\
\hline OWENS, M.R. & State government and Libraries & 1976 \\
\hline TAYLOR, R. S. & $\begin{array}{l}\text { Reminiscing about the Future: Professional } \\
\text { Education and the Information Environment }\end{array}$ & 1979 \\
\hline BREIVIK, P. & Putting libraries back in the information society & 1985 \\
\hline KUHLTHAU, C.C. & $\begin{array}{l}\text { Information skills for an information society: a } \\
\text { review of research. }\end{array}$ & 1987 \\
\hline $\begin{array}{l}\text { ALA. American Library } \\
\text { Association }\end{array}$ & $\begin{array}{l}\text { Report of the Presidential Committee on } \\
\text { Information Literacy: Final Report. }\end{array}$ & 1989 \\
\hline BEHRENS, S.J. & Librarians and Information Literacy & 1992 \\
\hline DOYLE, C. & $\begin{array}{l}\text { Information Literacy in an Information Society: a } \\
\text { concept for the information age }\end{array}$ & 1994 \\
\hline
\end{tabular}

Quadro 1 Autores fundadores da pesquisa sobre information literacy Fonte: Dudziak, 2001.

\section{ESTUDO BIBLIOMÉTRICO COMPARATIVO ENTRE AS BASES DE DADOS ISI WEB OF SCIENCE E SCOPUS}

A partir do panorama evolutivo traçado com relação às pesquisas sobre information literacy (competência informacional), o objetivo agora é analisar a produtividade científica em torno do tema em anos mais recentes (período de 2000 a 2010), tendo como referência a pesquisa realizada em duas bases de dados internacionais multidisciplinares dedicadas à análise de citações: ISI Web of Science (WoS) e SCOPUS. A partir de uma varredura realizada nas Bases mencionadas, a seguir são apresentados os resultados obtidos a partir das principais tipologias documentais (número de publicações sobre "information literacy" por tipo de publicação), autores mais citados, ranqueamento de periódicos que publicaram artigos sobre o tema, tipologia dos estudos, idiomas e autores mais citados na última década. O levantamento de informações foi feito entre os dias 13 e 14 de setembro de 2010.

\footnotetext{
${ }^{4}$ Não foi possível o acesso a este documento, embora esteja indicado nas Referências.
} 
Foi utilizada a versão on-line da Base de Dados Web of Science - WoS Science Citation Index Expanded e Social Science Citation Index; Arts \& Humanities Citation Index, bem como o Conference Proceedings Citation Index-Science. Como estratégia de busca no WoS foi utilizada a expressão "information literacy" como tópico, opção default do sistema.

Paralelamente, foi feito o mesmo levantamento na Base de Dados online Scopus, utilizando a expressão "information literacy" inicialmente recuperada no campo keyword. A Figura 1 apresenta as principais tipologias documentais relacionadas ao tema obtidas na Base WoS. A Figura 2 apresenta os dados obtidos na Base Scopus.

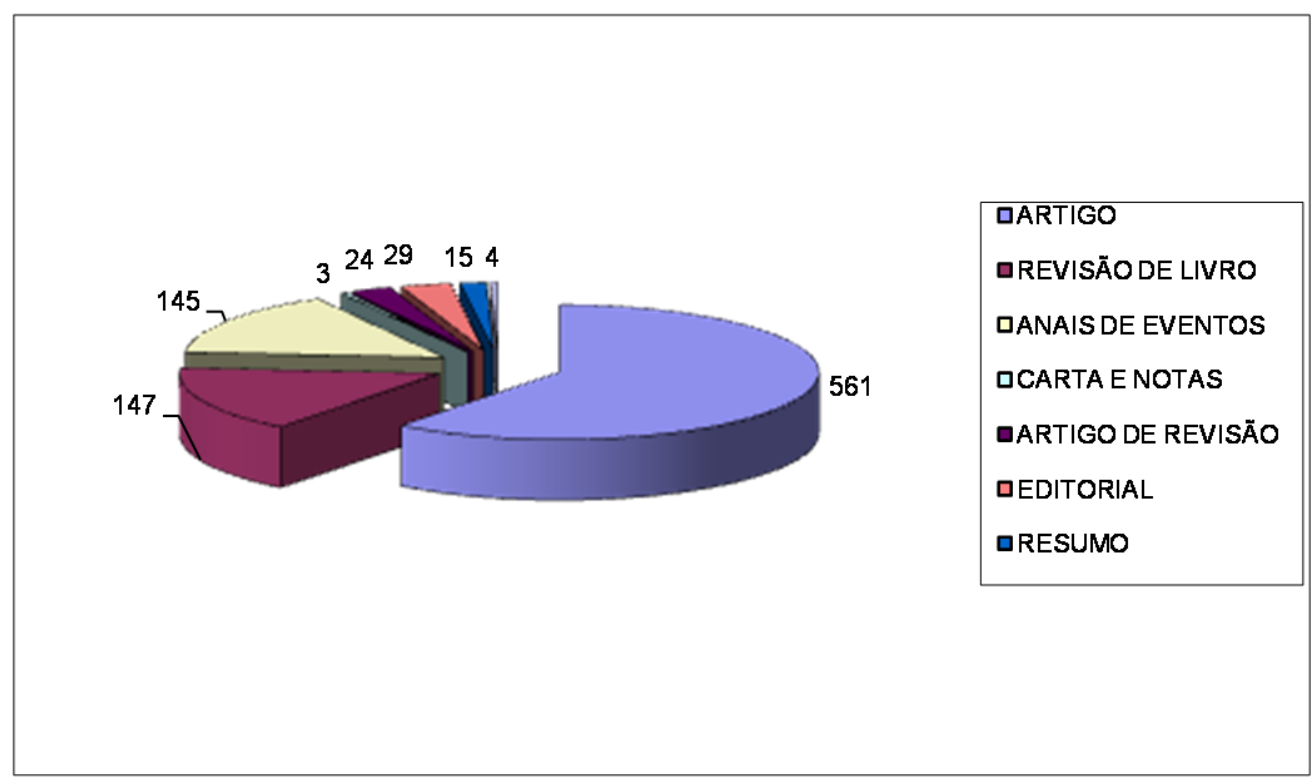

Figura 1 Número de publicações sobre "information literacy" por tipo de publicação (2000-Sept.2010)

Fonte: Web of Science, 2010 


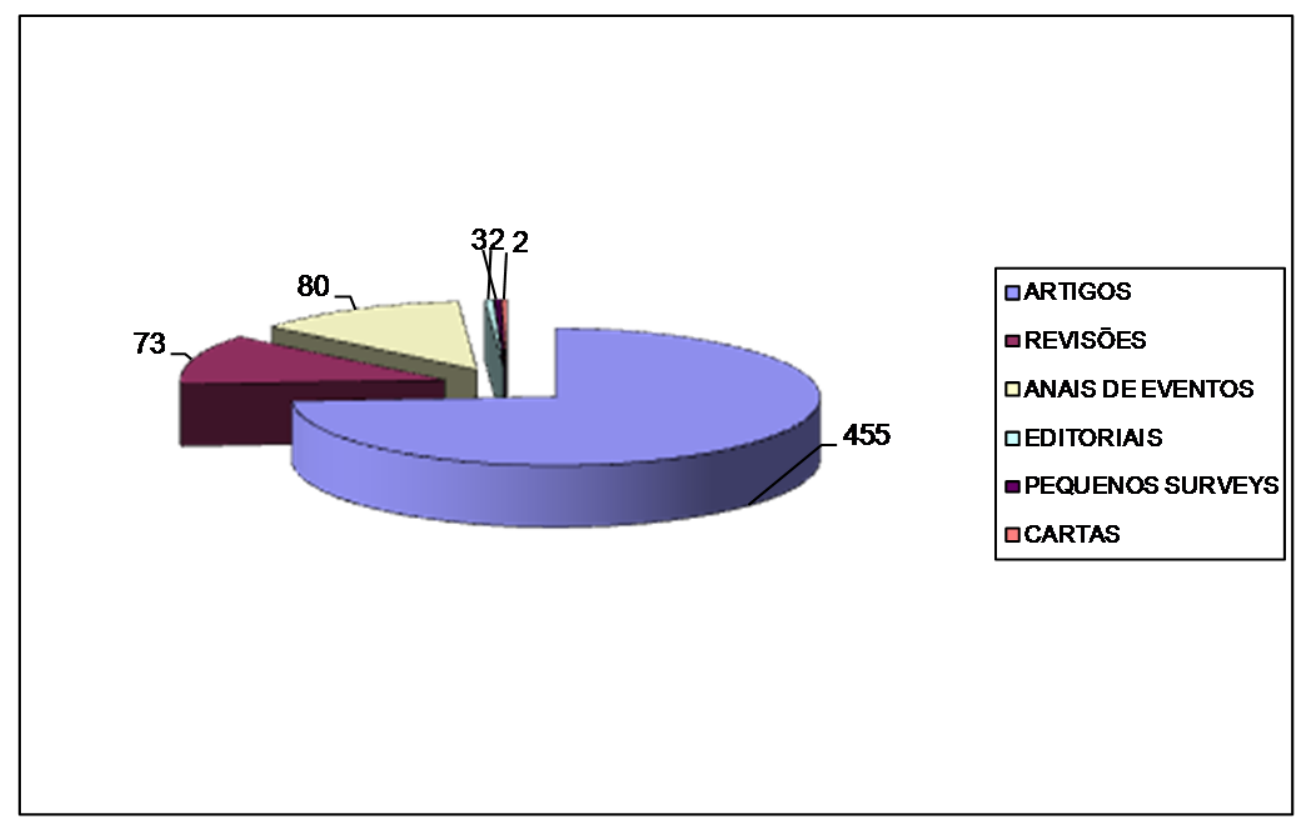

Figura 2 Número de publicações sobre "information literacy" por tipo de publicação (2000-Sept.2010)

Fonte: SCOPUS, 2010)

Enquanto na Web of Science foram recuperados 928 documentos, na Base Scopus foram levantados 615 documentos. Esta diferença tanto pode ser devida às diferenças de abrangência entre as Bases, quanto à forma como o próprio sistema estrutura a pesquisa. Tanto no WoS quanto no SCOPUS, observa-se clara prevalência dos artigos de periódicos (561 itens - WoS e 455 no Scopus), seguidos pela revisão de livros (147 itens no WoS e 73 no Scopus) e trabalhos apresentados em eventos (145 itens no WoS e 80 no Scopus).

A evolução da produção científica sobre o assunto (Base Scopus) está explicitada na Tabela 1 abaixo.

Tabela 1 Evolução da produção científica sobre o tema ao longo da década

\begin{tabular}{r|r|r|r|r|r|r|r|r|r}
\hline 2000 & 2001 & 2002 & 2003 & 2004 & 2005 & 2006 & 2007 & 2008 & 2009 \\
\hline 11 & 16 & 10 & 13 & 18 & 41 & 93 & 78 & 112 & 130 \\
\hline
\end{tabular}

Fonte: SCOPUS - 2000-Sept.2010)

Quanto às áreas de conhecimento, observa-se que a Base WoS oferece uma especificidade maior neste quesito, enquanto que a Base Scopus é mais superficial no tratamento dos dados, considerando apenas a grande área das Ciências Sociais. $\mathrm{Na}$ WoS, a pesquisa revelou uma clara tendência de publicação na Ciência da Informação (representando 52\% do total de publicações), corroborando a inequívoca ligação entre competência informacional e ciência da informação. Segue-se a área da Ciência da Computação e Sistemas de Informação com 22\% dos registros, e 9\% dos itens publicados na área da Educação. 

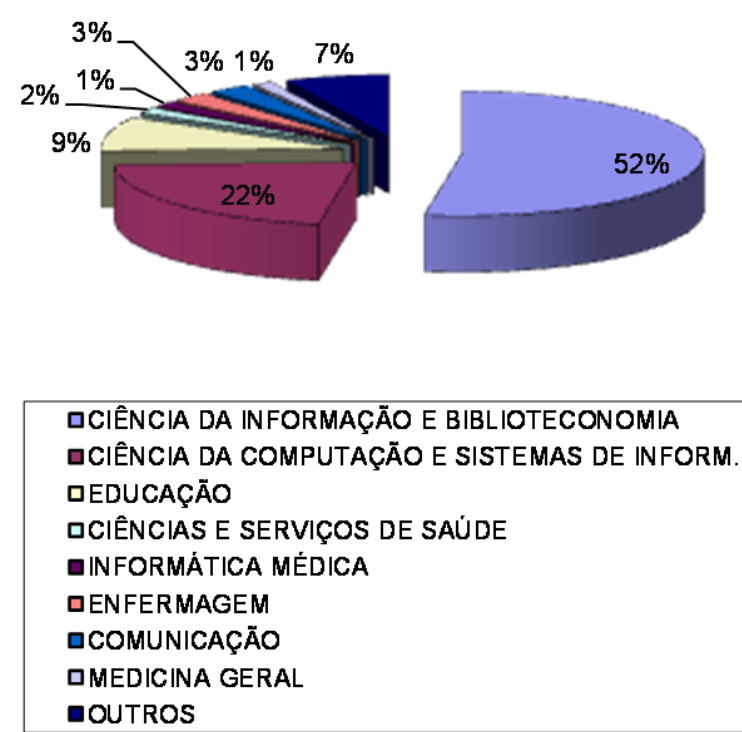

Figura 3 Porcentagem de Publicações sobre "information literacy" por Área de conhecimento (2000-Sept.2010)

Fonte: Web of Science, 2010

Já na Base Scopus, 58\% das publicações está na Área das Ciências Sociais, seguida da área da Ciência da Computação com 15\%, Negócios, Administração e Contabilidade (9\%), Engenharia (6\%), Medicina (6\%).

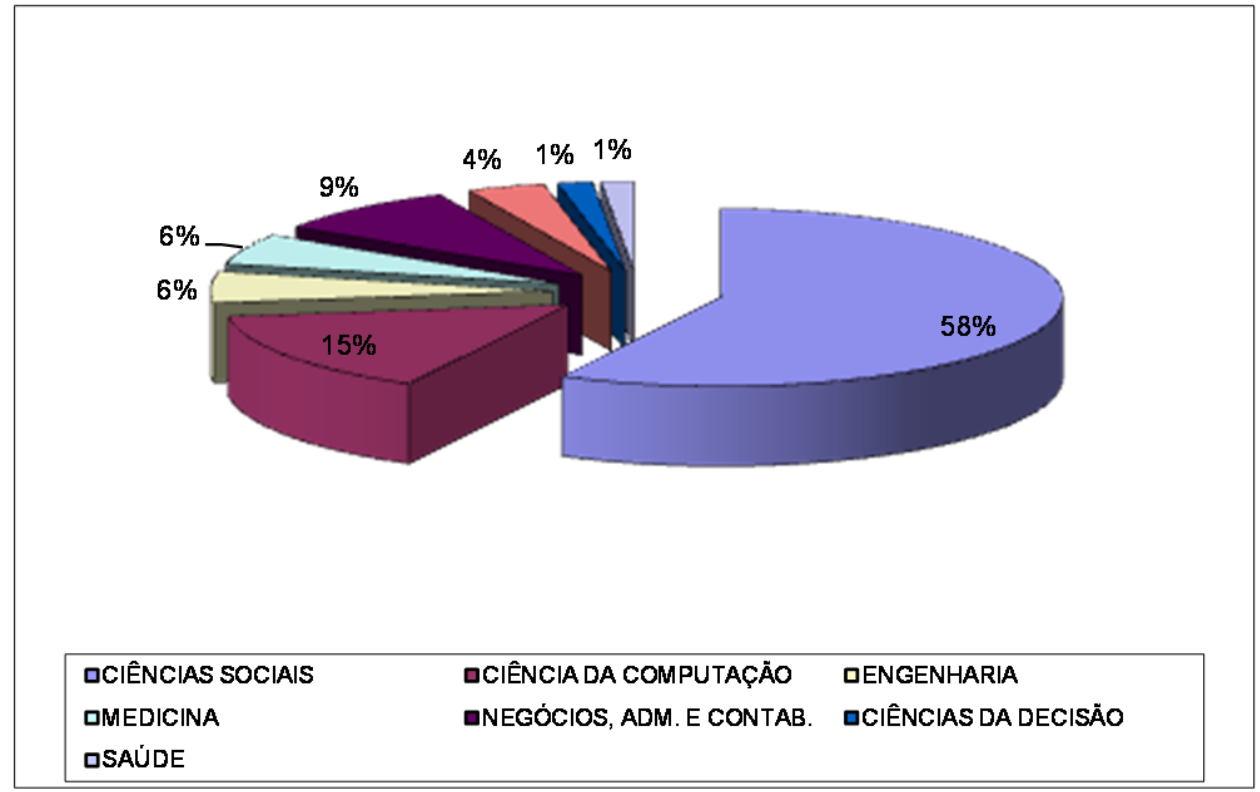

Figura 4 Porcentagem de Publicações sobre "information literacy" por Área de conhecimento (2000-Sept.2010)

Fonte: SCOPUS, 2010) 
Quanto ao idioma, a maioria dos documentos foi produzida em inglês, respondendo por cerca de $96 \%$ do total das publicações recuperadas tanto na WoS (896 artigos em inglês) quanto na Base Scopus (586 artigos em inglês). Observa-se a baixa ocorrência de publicações em espanhol (14 artigos na Base Wos e 16 artigos na Base Scopus) e português (9 artigos na Base WoS e 4 artigos na Base Scopus).

Quanto ao país de origem do documento, constata-se a predominância dos Estados Unidos, respondendo por mais da metade das publicações sobre o assunto (52\%), seguindo de modo distante pela Inglaterra, que responde por $9 \%$ das publicações. Ainda assim, dia a dia mais países se integram ao universo de pesquisadores. Tais dados só puderam ser obtidos na Base de Dados WoS (Fig. 5).

O núcleo de periódicos que mais publica sobre o tema varia de Base para Base, conforme dados apresentados a seguir (Fig. 6).

$\mathrm{Na}$ Base de dados WoS, este núcleo está restrito a cerca de dez títulos mais relevantes, embora se observe que o tema é explorado em distintos periódicos, em áreas do conhecimento diversas, somando 244 registros. Há um especial predomínio do periódico Journal of Academic Librarianship, com 95 artigos na década, seguido pelo Portal Libraries and The Academy com 56 artigos e o periódico College \& Research Libraries com 53 artigos. Os dados obtidos reforçam o predomínio de artigos e pesquisas produzidos na área da Ciência da Informação e Biblioteconomia.

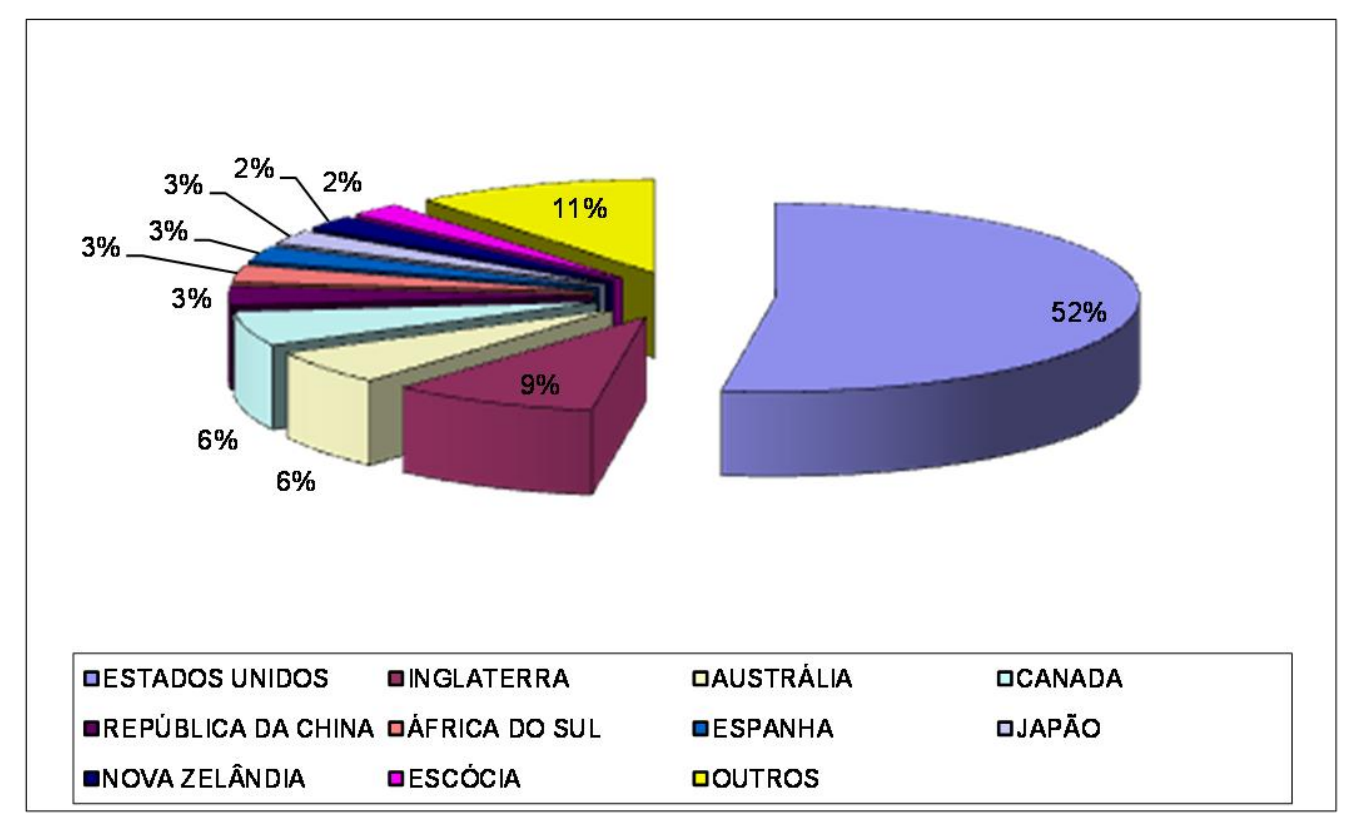

Figura 5 Número de publicações sobre "information literacy" por país (2000-Sept.2010)

Fonte: Web of Science, 2010 


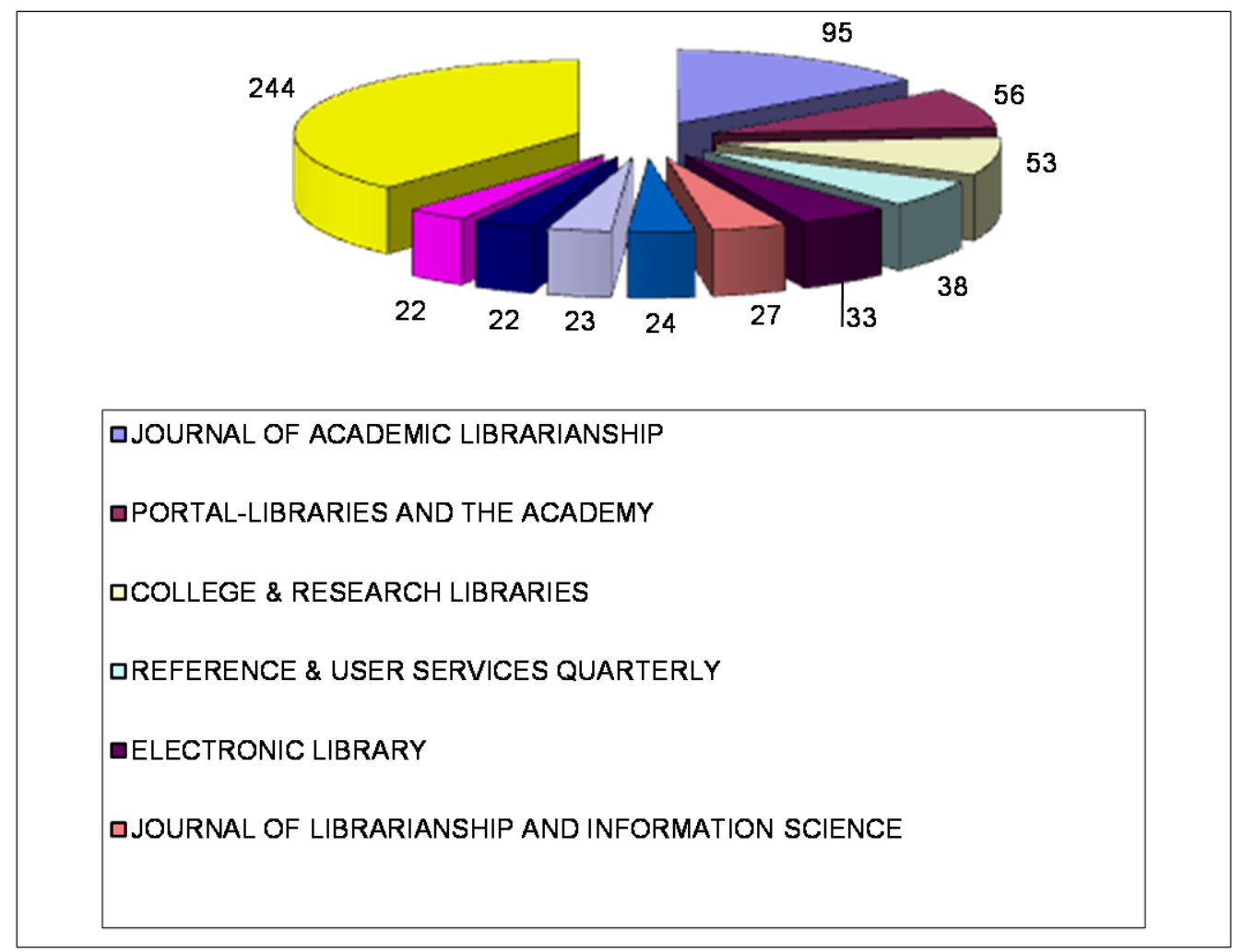

Figura 6 Principais Títulos de Periódicos que publicam artigos sobre "information literacy" (2000-Sept.2010)

Fonte: Web of Science, 2010)

Já na Base Scopus (Figura 7) o periódico que lidera o ranking é o Reference Services Review, com 69 artigos, seguido pelo College and Undergraduate Libraries, com 41 artigos sobre o tema. 


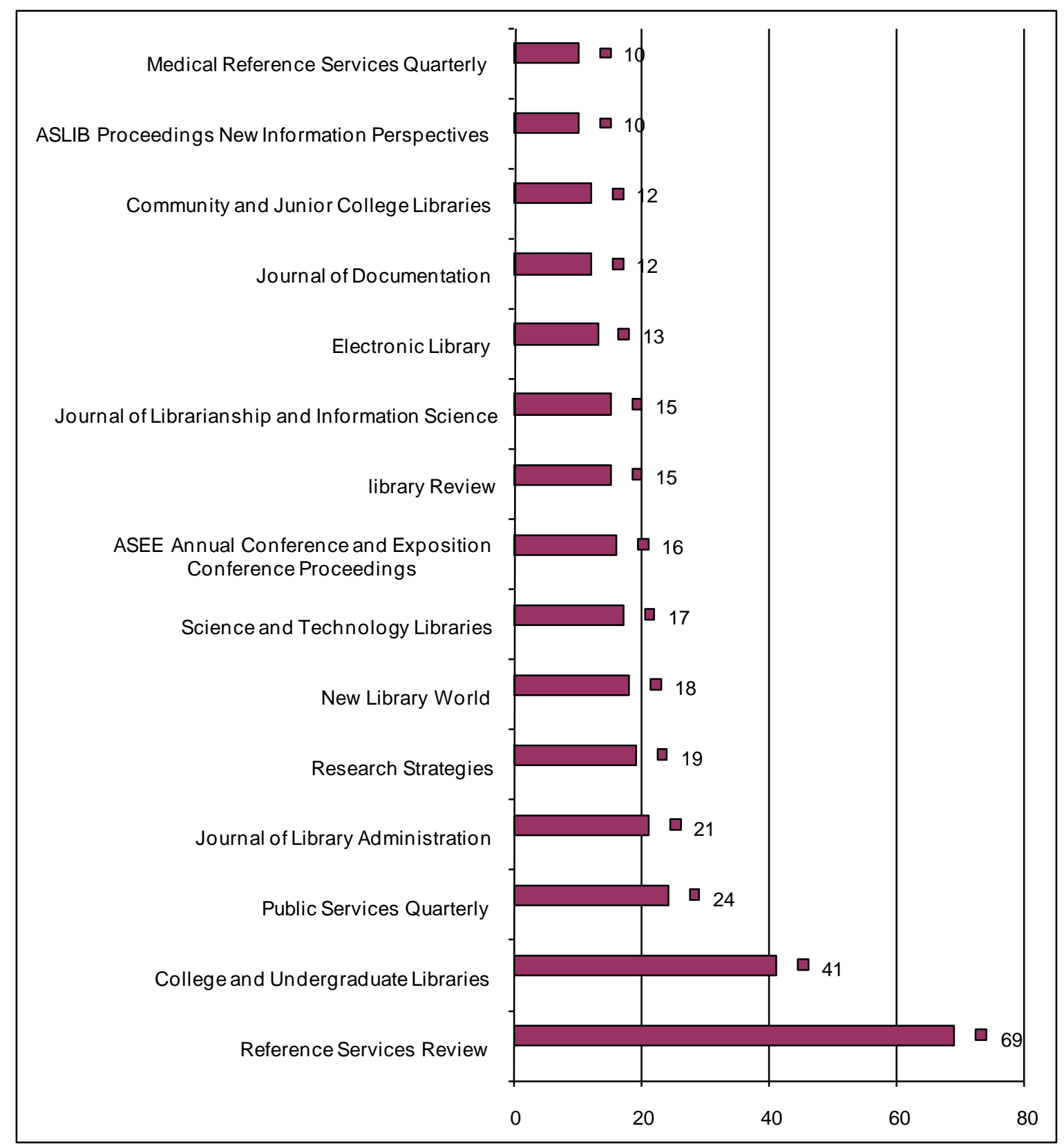

Figura 7 Principais títulos de periódicos que publicam artigos sobre "information literacy" (2000-Sept.2010)

Fonte: SCOPUS, 2010).

A seguir, são apresentados os artigos mais citados na última década (2000Sept. 2010) a respeito do tema, de acordo com a Base WoS, no Social Citation Report. 
Tabela 2 Ranking dos 25 artigos sobre "information literacy" mais citados (Período 2000-Sept.2010)

\begin{tabular}{|c|c|c|c|c|}
\hline Autor & Título do Artigo & Título do Periódico & Ano & Citações \\
\hline Hirsh, SG & Children's relevance criteria and information seeking on electronic resources & $\begin{array}{l}\text { JOURNAL OF THE AMERICAN SOCIETY } \\
\text { FOR INFORMATION SCIENCE }\end{array}$ & 1999 & 71 \\
\hline Bawden, D & Information and digital literacies: A review of concepts & JOURNAL OF DOCUMENTATION & 2001 & 66 \\
\hline Edmunds, A; Morris, A & $\begin{array}{l}\text { The problem of information overload in business organisations: a review of the } \\
\text { literature }\end{array}$ & $\begin{array}{l}\text { INTERNATIONAL JOURNAL OF } \\
\text { INFORMATION MANAGEMENT }\end{array}$ & 2000 & 54 \\
\hline Webber, S; Johnston, B & Conceptions of information literacy: new perspectives and implications & JOURNAL OF INFORMATION SCIENCE & 2000 & 48 \\
\hline Behrens, SJ & Conceptual analysis and historical overview of information literacy & COLLEGE \& RESEARCH LIBRARIES & 1994 & 38 \\
\hline Hart, A; Henwood, F; Wyatt, S & $\begin{array}{l}\text { The role of the Internet in patient-practitioner relationships: Findings from a } \\
\text { qualitative research study }\end{array}$ & $\begin{array}{l}\text { JOURNAL OF MEDICAL INTERNET } \\
\text { RESEARCH }\end{array}$ & 2004 & 32 \\
\hline Verhey, MP & $\begin{array}{l}\text { Information literacy in an undergraduate nursing curriculum: Development, } \\
\text { implementation, and evaluation }\end{array}$ & JOURNAL OF NURSING EDUCATION & 1999 & 32 \\
\hline $\begin{array}{l}\text { Fisher, KE; Durrance, JC; Hinton, } \\
\text { MB }\end{array}$ & $\begin{array}{l}\text { Information grounds and the use of need-based services by immigrants in } \\
\text { Queens, New York: A context-based, outcome evaluation approach }\end{array}$ & $\begin{array}{l}\text { JOURNAL OF THE AMERICAN SOCIETY } \\
\text { FOR INFORMATION SCIENCE AND } \\
\text { TECHNOLOGY }\end{array}$ & 2004 & 29 \\
\hline Foster, A & A nonlinear model of information-seeking behavior & $\begin{array}{l}\text { JOURNAL OF THE AMERICAN SOCIETY } \\
\text { FOR INFORMATION SCIENCE AND } \\
\text { TECHNOLOGY }\end{array}$ & 2004 & 28 \\
\hline Brennan, PF & $\begin{array}{l}\text { Health informatics and community health: Support for patients as collaborators in } \\
\text { care }\end{array}$ & $\begin{array}{l}\text { METHODS OF INFORMATION IN } \\
\text { MEDICINE }\end{array}$ & 1999 & 27 \\
\hline $\begin{array}{l}\text { McNeil, BJ; Elfrink, VL; Bickford, } \\
\text { CJ; Pierce, ST; Beyea, SC; Averill, } \\
\text { C; Klappenbach, C }\end{array}$ & $\begin{array}{l}\text { Nursing information technology knowledge, skills, and preparation of student } \\
\text { nurses, nursing faculty, and clinicians: A US survey }\end{array}$ & JOURNAL OF NURSING EDUCATION & 2003 & 27 \\
\hline Grafstein, A & A discipline-based approach to information literacy & $\begin{array}{l}\text { JOURNAL OF ACADEMIC } \\
\text { LIBRARIANSHIP }\end{array}$ & 2002 & 27 \\
\hline $\begin{array}{l}\text { Tuominen, K; Savolainen, R; Talja, } \\
\text { S }\end{array}$ & Information literacy as a sociotechnical practice & LIBRARY QUARTERLY & 2005 & 26 \\
\hline Leckie, GJ; Fullerton, A & $\begin{array}{l}\text { Information literacy in science and engineering undergraduate education: Faculty } \\
\text { attitudes and pedagogical practices }\end{array}$ & COLLEGE \& RESEARCH LIBRARIES & 1999 & 26 \\
\hline Elmborg, J & Critical information literacy: Implications for instructional practice & $\begin{array}{l}\text { JOURNAL OF ACADEMIC } \\
\text { LIBRARIANSHIP }\end{array}$ & 2006 & 24 \\
\hline Dunn, K & $\begin{array}{l}\text { Assessing information literacy skills in the California State University: A progress } \\
\text { report }\end{array}$ & $\begin{array}{l}\text { JOURNAL OF ACADEMIC } \\
\text { LIBRARIANSHIP }\end{array}$ & 2002 & 24 \\
\hline Marcum, JW & Rethinking information literacy & LIBRARY QUARTERLY & 2002 & 24 \\
\hline $\begin{array}{l}\text { Jacobs, SK; Rosenfeld, P; Haber, } \\
\text { J }\end{array}$ & $\begin{array}{l}\text { Information literacy as the foundation for evidence-based practice in graduate } \\
\text { nursing education: a Curriculum-integrated approach }\end{array}$ & JOURNAL OF PROFESSIONAL NURSING & 2003 & 22 \\
\hline Snavely, L; Cooper, N & The information literacy debate & $\begin{array}{l}\text { JOURNAL OF ACADEMIC } \\
\text { LIBRARIANSHIP }\end{array}$ & 1997 & 22 \\
\hline
\end{tabular}

Fonte: Web of Science, Sept.2010. 
Com relação à produção científica sobre o tema no Brasil, referente aos dois últimos anos (2008-2010), a representatividade nas Bases de dados utilizadas é baixa (onze artigos identificados na WoS e seis artigos identificados no Scopus, cuja origem é brasileira). As referências destes artigos são apresentadas a seguir.

Title: From reference work to information literacy: evolution of the librarians' educational role Author(s): Campello B.

Source: INVESTIGACION BIBLIOTECOLOGICA Volume: 24 Issue: 50 Pages: 83-108 Published: JANAPR 2010 Times Cited: 0

Title: The information literacy and library PUC-Campinas graduation: an analysis of the 2008

Author(s): Nascimento LD, Beraquet VSM

Source: PERSPECTIVAS EM CIENCIA DA INFORMACAO Volume: 14 Issue: 3 Pages: $2-19$ Published:

SEP-DEC 2009 Times Cited: 0

Title: Information literacy and dimensions of the competence of the librarian in the school context Author(s): Farias CM, Vitorino EV

Source: PERSPECTIVAS EM CIENCIA DA INFORMACAO Volume: 14 Issue: 2 Pages: 2-16 Published: MAY-AUG 2009 Times Cited: 0

Title: LIBRARIES IN DISTANCE EDUCATION: if the Consortium CEDERJ

Author(s): Filha MHFM, Cianconi RD

Source: INFORMACAO \& SOCIEDADE-ESTUDOS Volume: 20 Issue: 1 Pages: 129-138 Published: 2010 Times Cited: 0

Title: Where is the wisdom? I - A conceptual history of evidence-based medicine

Author(s): Wyer PC, Silva SA

Source: JOURNAL OF EVALUATION IN CLINICAL PRACTICE Volume: 15 Issue: 6 Pages: 891-898

Published: DEC 2009 Times Cited: 3

Title: BEACONS OF INFORMATION SOCIETY: a critical analysis about information literacy situation in Brazil Author(s): Dudziak EA

Source: INFORMACAO \& SOCIEDADE-ESTUDOS Volume: 18 Issue: 2 Pages: 41-53 Published: 2008 Times Cited: 0

Title: DIGITAL LITERACY: methodological parameters for informational skill

Author(s): de Pontes J, Talamo MDGM

Source: INFORMACAO \& SOCIEDADE-ESTUDOS Volume: 19 Issue: 2 Pages: 81-97 Published: 2009

Times Cited: 0

Title: Information literacy and evidence-based medicine

Author(s): Evangelista R, de Oliveira VDF, Pereira SL, et al.

Source: TRANSINFORMACAO Volume: 20 Issue: 1 Pages: 73-81 Published: 2008 Times Cited: 0

Title: Participative construction of a public policy instrument for information management and access Author(s): Freire GHD

Source: PERSPECTIVAS EM CIENCIA DA INFORMACAO Volume: 13 Issue: 3 Pages: 195-207 Published: SEP-DEC 2008 Times Cited: 0

Title: Citizenship and work in the information society: an approach based on the Information Literacy Author(s): dos Santos EM, Duarte EA, Prata NV

Source: PERSPECTIVAS EM CIENCIA DA INFORMACAO Volume: 13 Issue: 3 Pages: 208-222

Published: SEP-DEC 2008 Times Cited: 0

Title: Citizenship and work in the information society: an approach based on the Information Literacy Author(s): dos Santos EM, Duarte EA, Prata NV

Source: PERSPECTIVAS EM CIENCIA DA INFORMACAO Volume: 13 Issue: 3 Pages: 208-222

Published: SEP-DEC 2008 Times Cited: 0

\section{Quadro 2 Artigos brasileiros sobre "information literacy" publicados entre 2008-2010 e recuperados nas Bases de Dados WoS e Scopus (Sept. 2010)}


Alterando os parâmetros para obtenção de dados apenas para os autores que mais foram citados ou escreveram artigos sobre o tema de 2008 a 2010, considerando-se o tópico selecionado (information literacy), observa-se um número distinto de autores e citações recorrentes. Padrão semelhante também está presente no levantamento realizado na Base de Dados Scopus. Destacam-se os estudos de Heide Julien da University of Alberta, School of Library and Information Studies, Edmonton (Canada), Annemaree Lloyd da Charles Sturt University, School of Information Studies, (Australia), e Maria Pinto da Universidad de Granada, Department of Information Science, Granada, (Espanha). É preciso salientar ainda a recente e excelente revisão feita por Pinto, Cordon e Gómez Diaz (2010) sobre o tema, abrangendo o período de 1977 a 2007.

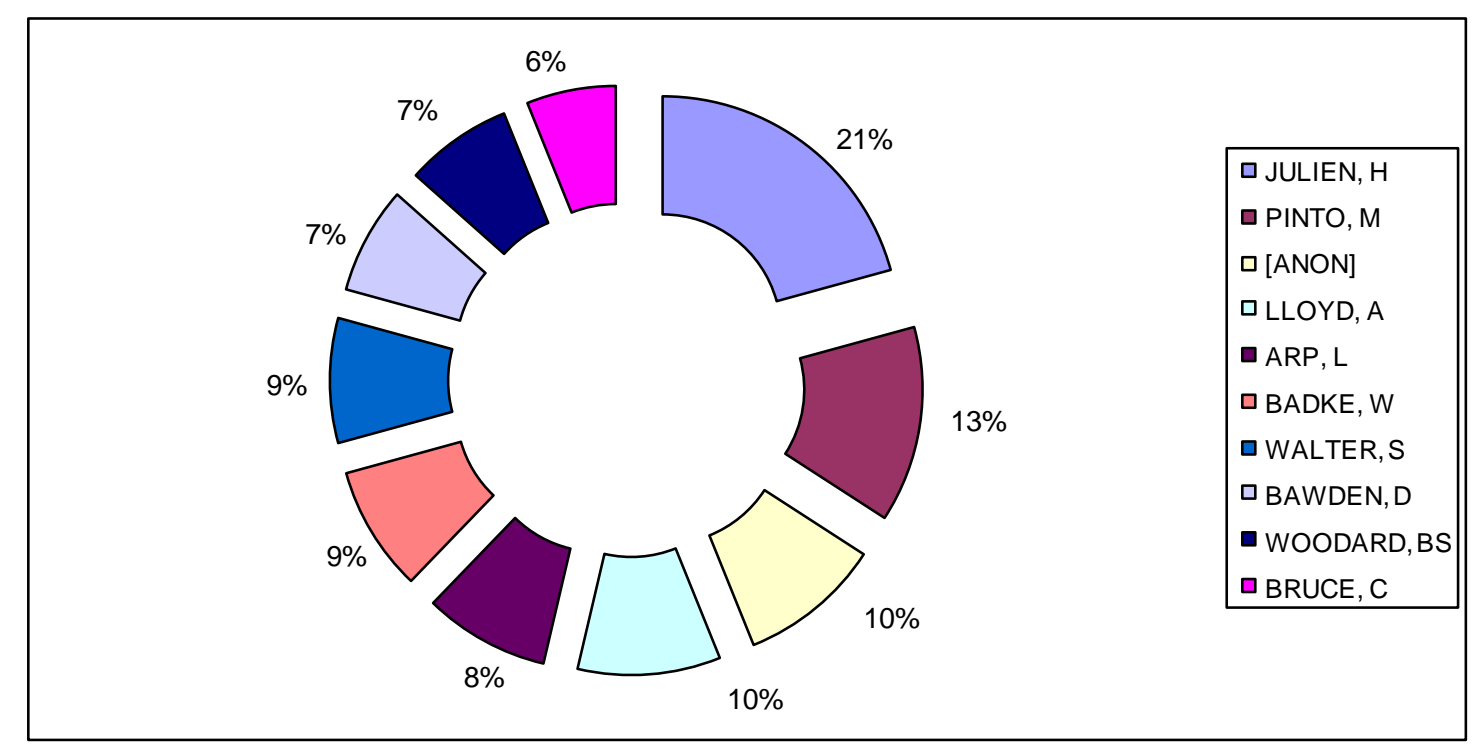

Figura 8 Autores mais citados relacionados ao tópico (2008-Sept. 2010) Fonte: Web of Science, 2010

\section{CONSIDERAÇÕES FINAIS}

Observa-se que os resultados obtidos nas pesquisas realizadas nas bases de dados selecionadas são distintos, tanto em número quanto em relação aos periódicos mais relevantes e autores mais citados, o que sugere cautela na interpretação dos resultados, dado que as fontes de informação variam. Apesar destas distinções, observa-se que há um núcleo específico de fontes de informação que delimitam uma área específica em torno da qual encontram-se agrupados os estudos e as pesquisas.

Do ponto de vista da abrangência dos estudos, a evolução na compreensão do conceito e suas relações com o dia a dia das pessoas conduziu a modificações que hoje se refletem tanto na produção científica quanto nas práticas educacionais, bem como no modo como a competência em informação (information literacy) é abordada pelos autores. 
Desde os anos 90, a information literacy disseminou-se em centenas de países, fazendo com que a produtividade científica na área crescesse exponencialmente. Porém, isso não significou necessariamente uma mudança no fazer bibliotecário, muitas vezes limitado ao espaço de atuação na biblioteca. Observa-se, entretanto, que o significado subjacente à expressão information literacy necessariamente rompe as fronteiras entre a Biblioteca e o 'mundo informacional', necessariamente mais amplo.

A desterritorialização e conseqüente perda da 'segurança' profissional antes proporcionada pelo superado 'espaço' informacional limitado à Biblioteca, ainda assusta muitos bibliotecários, que não compreendem a real ruptura paradigmática envolvida. Mas é fato que a busca pela informação atualmente vai (e muito) além do uso da biblioteca e seus recursos.

Temas como mobilidade, convergência, web social, acesso livre à informação, semântica, realidade aumentada e tantas novas frentes de pesquisa têm exercido profunda influência sobre as atividades relacionadas à Ciência da Informação e à Competência em Informação.

As relações com a qualidade de vida (expressa por meio da competência informacional em saúde, em finanças, para a governança e cidadania, em educação, e tecnologia), aproximaram e expandiram a possibilidade de aplicação e sua apropriação por pessoas comuns, além dos profissionais e cientistas (HIGH-LEVEL, 2006). Compreender estes fenômenos é, agora, uma prioridade para organismos internacionais como a IFLA e a UNESCO.

De modo geral, os estudos e pesquisas abrangem hoje as ações relativas à promoção da competência em informação em distintos 'espaços': educacional, profissional (mundo do trabalho), cidadão. Os padrões ainda são importantes, mas não essenciais, dado que a contextualização de ações é o fator primordial para 0 sucesso da implementação de programas voltados à competência em informação. Neste sentido, conhecer o público alvo, suas necessidades e motivações tornaramse uma prioridade.

Ainda assim, persistem barreiras à plena compreensão e apropriação do conceito. A começar pela falta de consenso quanto à terminologia e significado. Embora a linha de pesquisa esteja plenamente e vigorosamente consolidada nos Estados Unidos, como atestam os resultados obtidos, em muitos outros países a institucionalização do tema ainda é precária, as ações são fragmentadas, a maior parte dos projetos pedagógicos não contempla o tema, existindo uma fragmentação de interesses e iniciativas que são, em geral, isoladas.

Apesar destes problemas, a competência em informação é um tema que tem mobilizado profissionais de várias áreas: bibliotecários, professores, administradores, educadores, pedagogos, jornalistas, políticos, médicos, engenheiros, etc. Por ser um assunto que permeia todo e qualquer processo de aprendizado, investigação, criação, resolução de problemas e tomada de decisão, a competência em informação transbordou as fronteiras da biblioteconomia e transformou-se em um movimento transdisciplinar mundial, como atestam os resultados obtidos nesta pesquisa. 
A presença de estudos e pesquisas oriundos do Brasil ainda é pequena nas bases estudadas. Entretanto, é preciso considerar que as bases de dados utilizadas reúnem por si mesmas, um núcleo de periódicos e outras publicações que não explicita o universo total dos periódicos nos quais artigos brasileiros sobre o tema estão sendo ou foram efetivamente produzidos.

Deste ponto de vista, torna-se muito importante potencializar a visibilidade das pesquisas e estudos conduzidos no Brasil, de modo a trazer ao mundo a real dimensão das atividades científicas desenvolvidas sobre o tema no território nacional.

\section{REFERÊNCIAS}

ALA. American Library Association. Presidential Committee on Information Literacy: Final Report. 1989. Disponível em: <http://www.ala.org/ala/mgrps/divs/acrl/ publications/whitepapers/presidential.cfm > Acesso em: 12 ago. 2010.

ALVARADO, R. U. A frente de pesquisa na literatura sobre a produtividade dos autores. Encontros Bibli: Revista Eletrônica de Biblioteconomia e Ciência da Informação, Florianópolis, v. 14, n. 28, p. 39-57, 2009.

BARRETO, A. A. Mudança estrutural no fluxo do conhecimento: a comunicação eletrônica. Ciência da Informação, Brasília, v. 27, n. 2, p. 122-127, maio/ago. 1998.

BEHRENS, S. J. Librarians and Information Literacy. Mousaion, Pretoria, v. 10, n. 1, 1992.

. A conceptual analysis and historical overview of information literacy. College and Research Libraries, Chicago, v. 55, n. 4, p. 309-322, 1994.

BLESSINGER, K.; HRYCAJ, P. Highly cited articles in library and information science: an analysis of content and authorship trends. Library \& Information Science Research, Norwood, v. 32, n. 2, p. 156-162, 2010.

BREIVIK, P. S. Putting libraries back in the information society. American Libraries, Chicago, v. 16, n. 1, 1985.

BURCHINAL, L. G. The communications revolution: America's third century challenge. In the future of organizing knowledge. In: TEXAS A \& M UNIVERSITY LIBRARY'S CENTENNIAL ACADEMIC ASSEMBLY, 1976. Proceedings. Arizona: Texas University, 1976.

DOYLE, C. S. Information Literacy in an Information Society: a concept for the information age. New York: Syracuse University, 1994. 
DUDZIAK, E. A. A Information literacy e o papel educacional das bibliotecas. 2001. 173 p. Dissertação (Mestrado em Ciências da Comunicação) - Escola de Comunicações e Artes da Universidade de São Paulo, São Paulo, 2001.

- Information literacy: princípios, filosofia e práticas. Ciência da Informação, Brasília, v. 32, n. 1, p. 23-35, jan./ abr. 2003. Disponível em: <http://revista.ibict.br/ index.php/ciinf/article/view/123/104> Acesso em: 12 set. 2010.

HAMELINK, C. An alternative to news. Journal of Communication, New York, v. 26, n. 4, p. 120-123, 1976.

HIGH-LEVEL Colloquium on Information Literacy and Lifelong Learning Final Report. Alexandria: UNESCO/NFIL/IFLA, 2006. Disponível em: <http://archive.ifla.org/III/ wsis/High-Level-Colloquium.pdf> Acesso em: 23 fev. 2011.

KUHLTHAU, C. C. Information skills for an information society: a review of research. Syracuse, NY: Syracuse University, 1987.

OWENS, M. R. State government and Libraries. Library Journal, New York, v. 101, p. 19-26, 1976.

PINHEIRO, L. V. R. Lei de Bradford: uma reformulação conceitual. Ciência da Informação, Brasília, v. 12, n.2, p. 59-80, jul./dez. 1983.

PINTO, A. L.; IGAMI, M. P. Z.; BRESSIANE, J. C. Visibilidade e monitoramento científico na área nuclear e ciências relacionadas: uma perspectiva a partir da produtividade do IPEN-CNEN/SP. Perspectivas em Ciência da Informação, v. 15, n. 2, p. 198-218, maio./ago. 2010. Disponível em: <http://portaldeperiodicos.eci. ufmg.br/index.php/pci/article/viewFile/1033/741> Acesso em: 12 set. 2010.

PINTO, M.; CORDÓN, J. A. GOMÉZ DIÁZ, R. Thirty years of information literacy (1977--2007: A terminological, conceptual and statistical analysis. Journal of Librarianship and Information Science, v. 42, n. 1, p. 3-19, mar. 2010.

PRITCHARD, A. Statistical bibliography or bibliometrics? Journal of Documentation, London, v. 25, n. 4, p. 348-49, Dec. 1969.

SILVEIRA, M. A. A.; BAZI, R. E. R. As referências nos estudos de citação: algumas questões para discussão. DataGamaZero- Revista de Ciência da Informação, v. 10, n. 4, ago. 2009. Disponível em: <http://datagramazero.org.br/ago09/Art_04.htm> Acesso em: 12 set. 2010.

Redes de textos científicos na Ciência da Informação: análise cientométrica da institucionalização de um campo científico. DataGamaZero- Revista de Ciência da Informação, v. 9, n. 3, jun. 2008. Disponível em:<www.dgz.org.br/jun08/Art_01.htm> Acesso em: 12 set. 2010. 
TAYLOR, R. Reminiscing about the future: professional education and the information environment. Library Journal, New York, v.104, n.16, p.1871-1875, Sep.1979.

ZURKOWSKI, P. G. The Library Context and the Information Context: Bridging the Theoretical Gap. Library Journal, New York, v. 106, n. 13, p.1381-1384, July 1981.

. Information services environment relationships and priorities. Washington, D.C: National Commission on Libraries and Information Science, 1974. Disponível em: <http://www.eric.ed.gov/PDFS/ED100391.pdf> Acesso em: 12 set. 2010.

\title{
Title
}

Information literacy: evolutionary analysis of worldwide researches and scientific productivity

\begin{abstract}
The goal of this paper is to analyze the scientific production and research tendencies on information Literacy, based on a search conducted in two major international multidisciplinary databases dedicated to citation analysis: Web of Science (WoS) and SCOPUS. It addresses the bibliometrics analysis and its importance in determining trends in scientific activity and production. Then, the evolution of the concept of information literacy, since its inception in 1974 until 2010 is presented. The collection was identified by a search of the keyword "information literacy" in the above-mentioned databases. It concludes presenting the world trends in information literacy studies.
\end{abstract}

Keywords: Information literacy. Scientific productivity. Citation analysis. Web of Science; SCOPUS.

\section{Título}

Alfabetización en la información: análisis de la evolución de las tendencias de la investigación y la productividad científica en ámbito mundial

\section{Resumen}

Tiene como objetivo analizar la productividad científica e identificar las tendencias de investigación sobre el tema de la alfabetización en la información (information literacy), teniendo por base investigación realizada en dos de las principales bases de datos internacionales multidisciplinares dedicada al análisis de citaciones: Web of Science (WoS) y SCOPUS. Inicia abordando el significado del análisis bibliométrico y su importancia en la determinación de las tendencias de la actividad y producción científicas. Presenta la evolución del concepto de alfabetización informacional, desde su origen (en 1974) hasta 2010. Realiza la exploración en la producción científica registrada en forma de artículos y documentos que se encuentran en las referidas bases utilizando como estrategia de 
búsqueda la expresión "alfabetización informacional". Concluye con un análisis de las tendencias mundiales de la investigación sobre la competencia en información.

\section{Palabras-clave}

Competencia en Información. Producción científica. Análisis de citaciones. Web of Science. SCOPUS.

Recebido em: 08/11/2010

Aceito em: 11/02/2011 